\title{
Progress in Photocatalytic Degradation of Organic Wastewater by Graphene Binary Composites
}

\author{
Xue-qin Wang ${ }^{1}$, Qin $\mathrm{He}^{2, *}$ \\ ${ }^{1}$ Chongqing College of Architecture and Technology, Chongqing 401331, China \\ ${ }^{2}$ China West Normal University, Sichuan 637002, China
}

\begin{abstract}
Graphene composites not only have most advantages of graphene, but also have the characteristics of easy regulation, morphology and so on. Among many applications, graphene composite photocatalytic degradation of organic wastewater is one of the most likely graphene technologies. At present, the most studied graphene complexes include graphene monophyletic complex, graphene binary complex and graphene ternary complex. In this paper, the preparation of graphene binary complex and the research status of photocatalytic degradation of organic wastewater are summarized, and the existing problems are analyzed in order to provide a reference for the further research and application of graphene complex photocatalytic degradation of organic wastewater.
\end{abstract}

\section{Preparation and application of graphene and its complexes}

\subsection{Properties and preparation of graphene and its complexes}

Graphene, also known as monolayer graphite, is a honeycomb-like two-dimensional planar material formed by the hybridization of carbon atoms through $\mathrm{sp} 2$ electron orbitals and arranged in hexagons ${ }^{[1]}$. It is the thinnest two-dimensional nanomaterials known at present. The unique structure of graphene makes it have many excellent properties, such as good electrical conductivity(The velocity of electrons in graphene is about $1 / 300$ of the speed of light, much higher than that of electrons in ordinary conductors), good thermal conductivity ${ }^{[2]}$, high mechanical strength (Its strength is more than 100 times that of steel) ${ }^{[3]}$, strong adsorption ability(Because of its large surface area) and photoelectric conversion performance ${ }^{[4]}$.

In order to improve the properties of graphene, scientists and researchers have prepared a variety of graphene materials, including modified graphene materials and graphene composites. Although the modified graphene materials are more dispersed and active than single graphene, they can not give full play to the function of graphene. At present, the starting point and breakthrough point of the research and application of graphene materials is graphene composites ${ }^{[5]}$. The application of graphene is inseparable from the preparation of graphene and its composites. There are three main methods for preparing graphene complex, namely solution blending method, original polymerization method and melt blending method ${ }^{[6]}$.

\subsection{Research and application of graphene composites for sewage treatment}

The research of graphene complex for wastewater treatment mainly focuses on organic wastewater and heavy metal wastewater. According to the principle, the treatment of refractory organic wastewater by graphene complex can be divided into adsorption and catalytic degradation two types. Graphene complex shows super ability to adsorb organic pollutants with huge specific surface area, but there are still many shortcomings ${ }^{[7-8]}$, such as the toxicity of graphene complexes to animals and plants, the difficulty of separation and recovery from aqueous solution after adsorption of pollutants, the existence of regeneration and bad utilization performance is not good enough, which may cause serious secondary pollution to the environment ${ }^{[9-10]}$. The preparation process of graphene complexes is complicated and the production cost is high. The study of adsorption of organic pollutants is still in the stage of model pollutants, which is not suitable for large-scale batch production and practical application ${ }^{[11-12]}$.

Because graphene has excellent electron transport properties, it can be combined with photocatalytic materials to effectively exert the synergistic effect of the two and further enhance the photocatalytic properties of the composites ${ }^{[13]}$. Photocatalysis refers to the catalytic reaction of photocatalyst under the action of light, which can mineralize organic pollutants into simple inorganic substances such as $\mathrm{CO}_{2}$ and $\mathrm{H}_{2} \mathrm{O}^{[14]}$. Hence, the photocatalytic action of graphene complexes can realize the catalytic degradation of organic pollutants using nontoxic solar energy resources, avoiding secondary pollution to the environment, and is an economical,

$\overline{{ }^{*} \text { Corresponding author: hq88221@126.com }}$ 
simple, efficient and promising wastewater treatment method ${ }^{[15]}$. In this paper, the preparation and application of graphene binary photocatalytic complex are summarized. In general, the role of graphene materials in photocatalytic properties is mainly reflected in three aspects, its large specific surface area and enhanced adsorption capacity of composites, its high electron transfer ability (which can be used as electron acceptor to delay the recombination of electron-hole pairs) and enhance the photocatalytic activity.

\section{Preparation and application of graphene binary photocatalytic complex}

Graphene binary complexes mainly include graphene binary complexes, graphene oxide binary complexes and reduced graphene oxide binary complexes.

\subsection{Graphene binary complex}

Lui et al synthesis of graphene-encapsulated nanocomposites by solvothermal reaction, whose photocatalytic degradation Methyl blue activity is 1.49 times higher than commercial p25, When the graphene load is $5 \%$, The photocatalytic performance is the highest ${ }^{[16]}$. This is due to the excellent conductivity of graphene, promoted Methyl blue degradation.

Wang Xinzhi [14] synthesized graphene/ $\mathrm{TiO}_{2}$ nanotubes (GN-TNT) from commercial $\mathrm{TiO}_{2}$ nanoparticles as titanium source. When dyes and As(III) coexist, GN-TNT still have good visible light degradation efficiency for Methyl blue, Reactive Black 5 dyes and As(III) ions. However, compared with the single existence system, the degradation efficiency of pollutants in the coexistence system is reduced.

$\mathrm{Lv}$ Huimin et al [17] prepared graphene / $\mathrm{TiO}_{2}$ complex by sol-gel method with butyl titanate as titanium source. It has good photocatalytic effect. The decomposition rate is up to $95.24 \%$ under the condition of sunlight.

Chen lidong et al . ${ }^{[18]}$ reviewed the current preparation methods of $\mathrm{ZnO}$ composites with graphene and the current research status of photocatalytic degradation of pollutants such as rhodamine B, methylene blue and crystalline violet dyes, indicating that $\mathrm{ZnO}$-graphene nanocomposites materials exhibit significant advantages in photocatalytic applications. However, most of the studied pollutants are dyes, and the removal and mechanism of other organic pollutants and new refractory pollutants are less studied.

In addition to $\mathrm{TiO}_{2}$ and $\mathrm{ZnO}$, graphene can also form composites with other metal oxides and compounds as photocatalytic materials. At present, researchers have prepared $\mathrm{CdS}, \mathrm{ZnS}, \mathrm{WO}_{3}, \mathrm{CuO}, \mathrm{Mn}_{3} \mathrm{O}_{4}, \mathrm{Mn}_{2} \mathrm{O}_{3}$, $\mathrm{SnO}_{2}$ and other graphene photocatalytic complexes ${ }^{[19-22]}$.

\subsection{Graphene oxide (GO) binary complex}

Wang Zhao et al ${ }^{[23]}$ prepared $\mathrm{TiO}_{2} /$ graphene composites by hydrothermal method, The photocatalytic activity of Rhodamine B was significantly higher than that of $\mathrm{TiO} 2$ prepared under the same conditions.

Chen et al ${ }^{[24]}$ prepared $\mathrm{GO} / \mathrm{TiO}_{2}$ composites using $\mathrm{TiCl}(3)$ and graphene oxide (GO) as raw materials to study the degradation of methyl orange (MO) under visible light.

Zhang et al. ${ }^{[25]}$ found that the degradation efficiency of $\mathrm{GO}-\mathrm{TiO}_{2}$ complex to methyl orange is still high after several consecutive cycles under ultraviolet light, but the conversion rate of methyl orange decreases after each cycle, which is due to the formation of $\mathrm{TiO}_{2}$ agglomerated particles on both sides of the GO. This agglomeration results in a decrease in the contact area between $\mathrm{MO}$ molecules and $\mathrm{TiO}_{2}$ particles and a decrease in photodegradation efficiency. when the composite was exposed at room temperature for 4 weeks, it could restore its relatively stable photocatalytic degradation performance to $\mathrm{MO}$, but after 8 weeks, its degradation activity.

$\mathrm{Li}{ }^{[26]}$ synthesized high performance $\mathrm{ZnO} / \mathrm{GO}$ nanocomposites by simple chemical deposition, Under visible light, The photocatalytic degradation efficiency of organic dyes is obviously improved compared with $\mathrm{GO}$ and $\mathrm{ZnO}$ particles, and annealing in $\mathrm{N}_{2}$ gas can further improve the photocatalytic efficiency.

Duan Yuandong ${ }^{[27]}$ et al. used Hummers improved method to obtain the complex $\mathrm{CO}_{3} \mathrm{O}_{4} / \mathrm{GO}$, and investigated the effects of several inorganic salt ions on catalytic degradation polyvinyl alcohol in the presence of oxidant. The results show that the catalytic activity of $\mathrm{CO}_{3} \mathrm{O}_{4} / \mathrm{GO}$ is high, $\mathrm{H}_{2} \mathrm{PO}_{4}^{-}$can promote the degradation reaction of polyvinyl alcohol, and $\mathrm{As}$ the $\mathrm{pH}$ approaches neutral, the more favorable the reaction is.

Er et al. ${ }^{[28]}$ synthesized $\mathrm{TiO}_{2} /$ graphene composites, Used for photocatalytic degradation of simulated antibiotic wastewater, and the optimum treatment process conditions were determined.

Guo Sheng et al. proved that under visible light, And when the GO mass fraction is $5 \%$, The degradation rate of $\mathrm{GO}-\mathrm{FePO}_{4}$ composites to $\mathrm{RB}$ is 2.87 times that of pure $\mathrm{FePO}_{4}$ and can be reused ${ }^{[29]}$. Similarly, $\mathrm{Ag}_{3} \mathrm{PO}_{4}-\mathrm{GO}$ photodegradation rate of methyl orange was 1 times higher than that of pure $\mathrm{Ag}_{3} \mathrm{PO}_{4}$. The high photocatalytic activity and stability are mainly attributed to the rapid transfer of electrons from $\mathrm{Ag}_{3} \mathrm{PO}_{4}$ and $\mathrm{GO}^{[30]}$.

\subsection{Reduced graphene oxide (RGO) binary complex}

Zhang $^{[31]}$ prepared RGO-TiO(2)(P25) nanocomposites by simple one-step hydrothermal method. Compared to pure p25 and p25-CNTs with the same carbon content, During the MB treatment, the reaction rate of the photocatalyst was obviously improved.

$\mathrm{Yan}^{[32]}$ proposed a novel microwave-assisted in situ growth method to prepare $\mathrm{RGO}^{-\mathrm{BiVO}_{4}}$ composite photocatalyst, The photodegradation efficiency of ciprofloxacin is much higher than that of pure $\mathrm{BiVO}_{4}$. 
especially when the mass content is $2 \% \mathrm{RGO}-\mathrm{BiVO}_{4}$ the ciprofloxacin degradation rate of composite photocatalyst is the highest $(68.2 \%$ within $60 \mathrm{~min})$. The improvement of photocatalytic activity is mainly due to the effective separation of electron hole pairs, rather than improving the light absorption.

Chen et al ${ }^{[33]}$ synthesized $\mathrm{ZnO} / \mathrm{RGO}$ composites by simple solvothermal reaction with obvious photocatalytic activity. Zinc vacancies and oxygen vacancies produced on the $\mathrm{ZnO}$ surface, which play a crucial role in the photocatalytic performance of the $\mathrm{ZnO}$. In the photodegradation process of methylene orange, both zinc and oxygen vacancies can cause effective charge separation, which can obviously inhibit charge recombination.

$\mathrm{Wu}$ Zhimin ${ }^{[34]}$ prepared visible light responsive BiOCl-RGO composites with stable performance and enhanced catalytic activity, and analyzed the kinetic model of photocatalytic degradation of Rhodamine B and the reasons for the enhancement of catalytic activity. on the one hand, $\mathrm{BiOCl}$ can prevent $\mathrm{RGO}$ agglomeration and improve the adsorption of dyes. on the other hand, the adsorbed dyes can be degraded by visible light photocatalysis, which makes BiOCl-RGO composites free from recycling and suitable for industrial applications.

$\mathrm{Lv}$ et $\mathrm{a}^{[35]}$. synthesized RGO modified ultralong $\mathrm{TiO}_{2}$ nanotubes (LTNTs) by improved hydrothermal method and heating reflux method. Under visible light irradiation, the photocatalytic activity of the composites is higher than that of pure TNT, which also confirms that the RGO in the composites play an important role in making more effective use of sunlight and improving the separation efficiency of electron hole pairs.

\section{Existing problems and prospects}

- Because of the hydrophobicity and agglomeration tendency of graphene, graphene nanomaterials are easy to aggregate, which affects the photocatalytic performance of composites ${ }^{[36]}$. The defects of the carbon atomic structure of graphene materials reduced by graphite oxide often lead to the decline of the properties of graphene materials.Therefore, the relationship between the preparation, structure and properties of graphene materials needs further study ${ }^{[37]}$.

- The ffects of graphene materials on the environment needs further study ${ }^{[37]}$. Development of graphene preparation technology with excellent performance, environment friendly and expandable production to provide guarantee for subsequent application research

- The photocatalytic degradation mechanism and degradation process of graphene composites to organic pollutants need to be further studied. At present, the laboratory degradation simulation wastewater is the main research, which is different from the actual wastewater quality, and the effect is not ideal in the actual water treatment application ${ }^{[38]}$.

- How to effectively treat and reuse graphene composites after degrading pollutants needs further study, which restricts the application of graphene composites [14].

\section{References}

1. Geim A K., Graphene: status and prospects. science, 324, 1530-1534, (2009)

2. Park S, Ruoff R S. Chemical methods for the production of graphenes, Nature Nanotechnology, 4, 217, (2009)

3. Young R J, Kinloch I A, Gong L, et al. The mechanics of graphene nanocomposites [J]: a review. Composites Science \& Technology, 72,1459-1476. (2012)

4. Bonaccorso, F Sun, Z. Hasan, et al. Graphene photonics and optoelectronics, Nature Photonics, 4, 611-612, (2010)

5. J.R.Chen, M Zhou, N Song, et al. Preparation and Application of Graphene Composites in Dye Wastewater Treatment, Proceedings of the 2015 Annual Conference of the Chinese Society of Environmental Sciences, 2, 2846, (2015)

6. Y. B. Liu, X. B. Zeng, C. M. Dai, et al. Advances in the Application of Graphene Nanocomposites in Water Treatment. Materials Reports, 7,128-129, (2013)

7. F. Liu, F. T. Fan, Y.C. Lv, et al. Research Progress on Adsorption and Removal of Hard-degradable Organics by Graphene and Its Composites. Acta Petrolei Sinica, 4, 863, (2016)

8. S. T. Yang, L. Q. Zhao, Advances in Preparation and Application of Graphene Adsorption Materials. Journal of Southwest Minzu University (Natural Science Edition), 2,216. (2014)

9. F Liu, F T Fan et al. Research Progress on Adsorption and Removal of Hard-degradable Organics by Graphene and Its Composites. Acta Petrolei Sinica (Petroleum Processing Section) 04, 863, (2016)

10. D Y Feng, Y R Sun et al. Adsorption of heavy metal ions in water by graphene and its composites. Jorunal of Functional Materials. 46,30093015(2015)

11. S N Sun,F Yu et al. Adsorption of organic matter and heavy metals in water by graphene and its composites. Modern Chemical Industry,11,35-36, (2015)

12. S T Tang, L Q Zhao et al. Advances in Preparation and Application of Graphene Adsorption Materials, Journal of Southwest Minzu University (Natural Science Edition),02,216, (2014)

13. Z. L. Hu, X. F.Li et al. Research of graphene materials in water treatment. Jorunal of Functional Materials, S1,3. 2016

14. X. Z. Wang, Preparation of graphene/titanium dioxide nanotube composite photocatalyst and its application in wastewater treatment. Thesis of Yantai University, (2014) 
15. Li J, Feng H, Jiang J, et al. One-pot in situ synthesis of a $\mathrm{CoFe} 2 \mathrm{O} 4$ nanoparticle-reduced graphene oxide nanocomposite with high performance for levodopa sensing. Rsc Advances, 5,121, 99669-99677, (2015)

16. Lui G, Liao J Y, Duan A, et al. Graphene-wrapped hierarchical $\mathrm{TiO}_{2}$ nanoflower composites with enhanced photocatalytic performance. Journal of Materials Chemistry A, 1, 12255-12262, (2013)

17. H. M. Lv, M. W. Tian, et al. Preparation and photocatalytic properties of graphene/titanium dioxide composite fabrics. Journal of Qingdao University (Engineering \& Technology Edition, 2,65-70. (2016)

18. L. D. Chen, J. X. Liu, et al. Advances in photocatalytic degradation of pollutants $\mathrm{ZnO}$ graphene composites, Industrial Wanter Treatment, 35,17-20. (2015)

19. Khan Z T Chetia $\mathrm{T} R$, Vardhaman A K, et al. Visiblelight assisted photocatalytic hydrogen generation and organic dye degradation by CdSmetal oxide hybrids inpresence of graphene oxide, RSC Advances, 2, 12122-12128, (2012)

20. Chandra S,Das $\mathrm{P}$, Bag S,et al. $\mathrm{Mn}_{2} \mathrm{O}_{3}$ decorated graphene nanosheet: An advanced material for the photocatalytic degradation of organic dyes, Materials Science and Engineering: B, 177,855-861. (2012)

21. Seema H, Kemp K C, Chandra V, et al. Graphene$\mathrm{SnO} 2$ composites for highly efficient photocatalytic degradation of methylene blue under sunlight. Nanotechnology, 23,355705, (2012)

22. Zhang J, Xiong Z, Zhao X S. Graphene-metaloxide composites for the degradation of dyes under visible light irradiation. Journal of Materials Chemistry, 21,3634-364, (2011),

23. Wang Zhao, Mao Feng, Huang Xiangping. Preparation and Photocatalytic activity of $\mathrm{TiO} 2$ /graphene composites, Materials Science and Engineering, 29 ,267, (2011)

24. Chen C, Cai W, Long M, et al. Synthesis of visiblelight responsive graphene oxide/ $\mathrm{TiO}_{2}$ composites with $\mathrm{p} / \mathrm{n}$ heterojunction. ACS Nano, 4, 6425-6432, (2010)

25. Zhang Q, He YQ, Chen XG, et al. Structure and photocatalytic properties of $\mathrm{TiO}_{2}$-graphene oxide intercalated composite. Chinese Science Bulletin, 56,331-339, (2011)

26. Li B, Liu $\mathrm{T}$, Wang $\mathrm{Y}$, et al. $\mathrm{ZnO}$ /graphene-oxide nanocomposite with remarkably enhanced visiblelight driven photocatalytic performance, Journal of Colloid \& Interface Science. 377,114-121, (2012)

27. Y.D. Duan, W. Lv, et al. Effects of inorganic salt ions on degradation of PVA wastewater $\mathrm{CO}_{3} \mathrm{O}_{4} / \mathrm{GO}$ supported catalysts. Chinese Journal of Environmental Engineering, 9, 4839-4844, (2015)

28. R. F. Er, W. Jiang, et al . Graphene/ $\mathrm{TiO}_{2}$ Treatment of Teramycin Hydrochloride Simulated Antibiotic Wastewater. Journal of Harbin University of
Commerce (Natural Sciences Edition), 3,337-340, (2016)

29. S. Guo, G. k. Zhang, et al. Enhanced photo-Fenton degradation of rhodamine $\mathrm{B}$ using graphene oxideamorphous FePO4 as effective and stable heterogeneous catalyst, Journal of Colloid and Interface Science, 448,460-466, (2015)

30. Z. 1. Xiu, Y. z. Wu, et al. Graphene oxide wrapped $\mathrm{Ag}_{3} \mathrm{PO}_{4}$ sub-microparticles with highly enhanced photocatalytic activity and stability under visible light irradiation, Materials Research Bulletin, 59,192-1988, (2014)

31. H. Zhang, X. Lv, et al. P25-graphene composite as a high performance photocatalyst. Acs Nano, 4,380386. (2010)

32. Yan Y, Sun S, Song Y, et al. Microwave-assisted in situsynthesis of reduced graphene oxide-BiVO4 composite photocatalysts and their enhanced photocatalytic performance for the degradation of ciprofloxacin, J Hazard material 250-251, 106-114. (2013)

33. T. T. Chen, I. C. Chang, et al. The exceptional photo-catalytic activity of $\mathrm{ZnO} / \mathrm{RGO}$ composite via metal and oxygen vacancies. Applied Catalysis B Environmental, s 142-143,442-449., (2013)

34. Z. M. Wu. Preparation and application of graphenebased adsorption and photocatalytic materials, Thesis of South China University of Technology, (2014)

35. S. Lv, J. Wan, et al. Preparation of superlong $\mathrm{TiO}_{2}$, nanotubes and reduced graphene oxide composite photocatalysts with enhanced photocatalytic performance under visible light irradiation, Journal of Materials Science Materials in Electronics, 1-8. (2017)

36. Jian-Ding Q, Guo-Chong W, Ru-Ping L, et al. Controllable Deposition of Platinum Nanoparticles on Graphene As an Electrocatalyst for Direct Methanol Fuel Cells. Journal of Physical Chemistry, 115,15639-15645, (2011)

37. Z. L. Hu, X. F. Li, et al. Research Progress of Graphene Materials in Water Treatment. Jorunal of Functional Materials, S1,1-6. (2016)

38. T. Fu, Application of Graphene Composites in Water Treatment. Environmental Science and Management, 02,111-114. (2017) 